\title{
Review of: "Characteristics of gut microbiota in people with obesity"
}

\author{
Mohamed Mannaa ${ }^{1}$ \\ 1 Cairo University
}

Potential competing interests: The author(s) declared that no potential competing interests exist.

The present research article "Characteristics of the gut microbiota in people with obesity" investigates the gut microbiome composition in obese subjects $(n=21)$ from Shandong Province, China. The metagenomic $16 \mathrm{~S}$ rRNA sequencing results were compared to healthy control subjects $(n=21)$ retrieved from the Microbial Genome Database System (http://data.mypathogen.org, ID = ICDC-20180224-143509) that were sequenced in a previous study from samples collected from Beijing, China.

Although such studies provide valuable knowledge about the links between gut microbiome and different types of disorders, one should raise some concerns about the current work:

- The fact that the control group was obtained from the available database of a different study and in a geographically different region might have affected the results, especially that the age category between the two groups is relatively high ( 35 and 26 years old in the obesity and control groups, respectively).

- One of the significant markers for the gut microbiome alterations with obesity that have been reported in most previous studies is the firmicutes/bacteroidetes ratio. In most studies adopting metagenomic tools to investigate the microbiome profile of obese and lean subjects, the firmicutes were consistently higher in the obesity group. However, in the current study, it is reported to be contrary to such findings as the firmicutes were higher in the control group $61 \%$ compared to obesity group $37 \%$. In contrast, the bacteroidetes were $32 \%$ and $53 \%$ in the control and obesity groups, respectively.

- $\quad$ The authors have supported such a result to only one study conducted in 2012 that was not even made using the same methodological approach "metagenomic (meta-barcoding) 16S rRNA". Moreover, the authors' justification of such dispute is that it "may be related to the region, environment and diet, for example, people in the Shandong Province of China prefer food made of flour". Yet, the authors contradicted such a statement at the end of the discussion section to justify the imitation of this study and the reason they ignored the differences between the geographical difference in both groups "Since both Jinan and Beijing are located in the north of China, with a distance of about $410 \mathrm{~km}$ and sharing the same environment and eating habits, we ignored the impact of geographical differences on the gut microbiota". Therefore, the rationale of this study needs to be revised!

- It was also noted from the numerical data represented in the table of relative abundance of microbial groups that the standard deviations in the obesity group were notably higher than the control group. This raises doubts on the compatibility of both sets of data and the possibilities of conducting a 
comparative analysis leading to sound conclusions.

- It is therefore strongly suggested that comparative analysis work should consider reducing the effect of other variables such as geographical location, sampling time, eating habit, age group, male/female ratios, etc.,

- $\quad$ For studying the functionality of the gut microbiome, although relying on the predictive metagenomics, such as the approach adopted in the current study, might be helpful to show the general direction based on the types of microbes, it would be more beneficial to integrate other available RNA or protein-based meta-omics and metabolomics tools for functional profiling.

- Another primary concern in the current paper is reporting the gut microbiome composition to the species level (abstract, Table 2, results, and conclusion). The method used for sequencing was based on meta-barcoding the 16S rRNA V3 and V4 variables, which doesn't provide enough depth to report the microbial taxonomy resolution to the species level. Therefore, it is recommended that such a method be used to convey the microbial taxonomy to the genus level (Johnson et al., 2019 Nature communications, 10).

- The correlation and links between certain microbes in the gut microbiome and the amount of fecal short-chain fatty acids (SCFAs) were highlighted in the discussion part "In our study, the concentration of Bacteroides uniformis is significantly reduced while Blautia and Prevotella copri are significantly increased in individuals with obesity (Table 2), indicating that fecal SCFAs in the Obesity group is increased." However, the level of fecal SCFAs was not evaluated, and its increase in the obesity group should not be stated as a fact without evaluation.

- Taken together, with the increasing evidence on the links between gut microbiome and obesity, it became possible to identify potential manipulation targets. This opens the way for future therapies based on modifying (correcting) the gut microbiome dysbiosis. Therefore, studies reporting comparisons between people with obesity to healthy subjects should consider the precise identification of obesity markers. One should do this by eliminating sources of bias or inaccurate results by careful selection of samples, sampling method, size and time, location, homogeneity, and other possible variables that might lead to misleading results. 